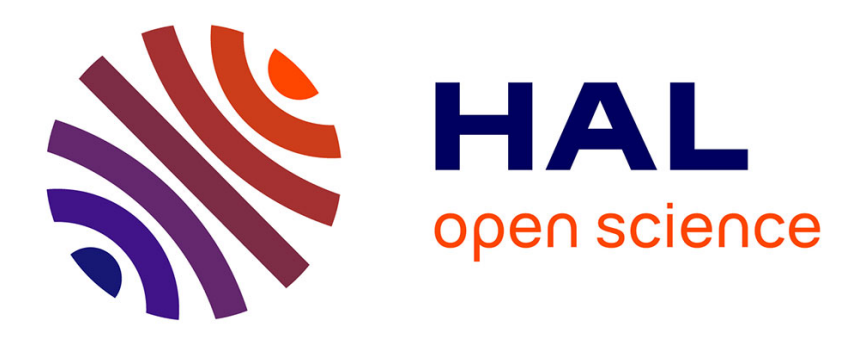

\title{
Conduite d'un Van de Graaff et des expériences par un calculateur
}

\author{
J.C. Bluet, J.L. Leroy, P. Fardeau
}

\section{To cite this version:}

J.C. Bluet, J.L. Leroy, P. Fardeau. Conduite d'un Van de Graaff et des expériences par un calculateur. Revue de Physique Appliquée, 1969, 4 (2), pp.129-130. 10.1051/rphysap:0196900402012900 . jpa00243177

\section{HAL Id: jpa-00243177 https://hal.science/jpa-00243177}

Submitted on 1 Jan 1969

HAL is a multi-disciplinary open access archive for the deposit and dissemination of scientific research documents, whether they are published or not. The documents may come from teaching and research institutions in France or abroad, or from public or private research centers.
L'archive ouverte pluridisciplinaire HAL, est destinée au dépôt et à la diffusion de documents scientifiques de niveau recherche, publiés ou non, émanant des établissements d'enseignement et de recherche français ou étrangers, des laboratoires publics ou privés. 


\title{
GONDUITE D'UN VAN DE GRAAFF ET DES EXPÉRIENGES PAR UN GALGULATEUR
}

\author{
J. G. BLUET, J. L. LEROY et P. FARDEAU, \\ Centre d'Études Nucléaires de Cadarache.
}

\begin{abstract}
Résumé. - Un calculateur CII 90-10 a été implanté auprès de l'accélérateur Van de Graaff $5 \mathrm{MeV}$ de Cadarache. L'énergie et les grandeurs directement liées sont contrôlées. En ce qui concerne les expériences, le système est chargé de l'acquisition des données et contrôle tous les paramètres. On insiste plus particulièrement sur les problèmes de programmation.

Abstract. - A CII 90-10 computer has been associated with the $5 \mathrm{MeV}$ Van de Graaff accelerator at Cadarache. Energy and directly related quantities are controlled. Concerning the experiments, the functions of the system are acquisition of data, and control of all the parameters. Software problems are more particularly discussed.
\end{abstract}

I. Composition du système [1]. - Le calculateur est du type 90-10 de la Compagnie Internationale pour l'Informatique, avec une mémoire de $32 \mathrm{k}$ et les périphériques suivants : télétype émettrice-réceptrice, lec- teur-perforateur de ruban, lecteur de cartes, imprimante rapide, deux unités de ruban magnétique et une table traçante Benson. L'équipement « temps réel » comprend 32 lignes d'entrée numérique

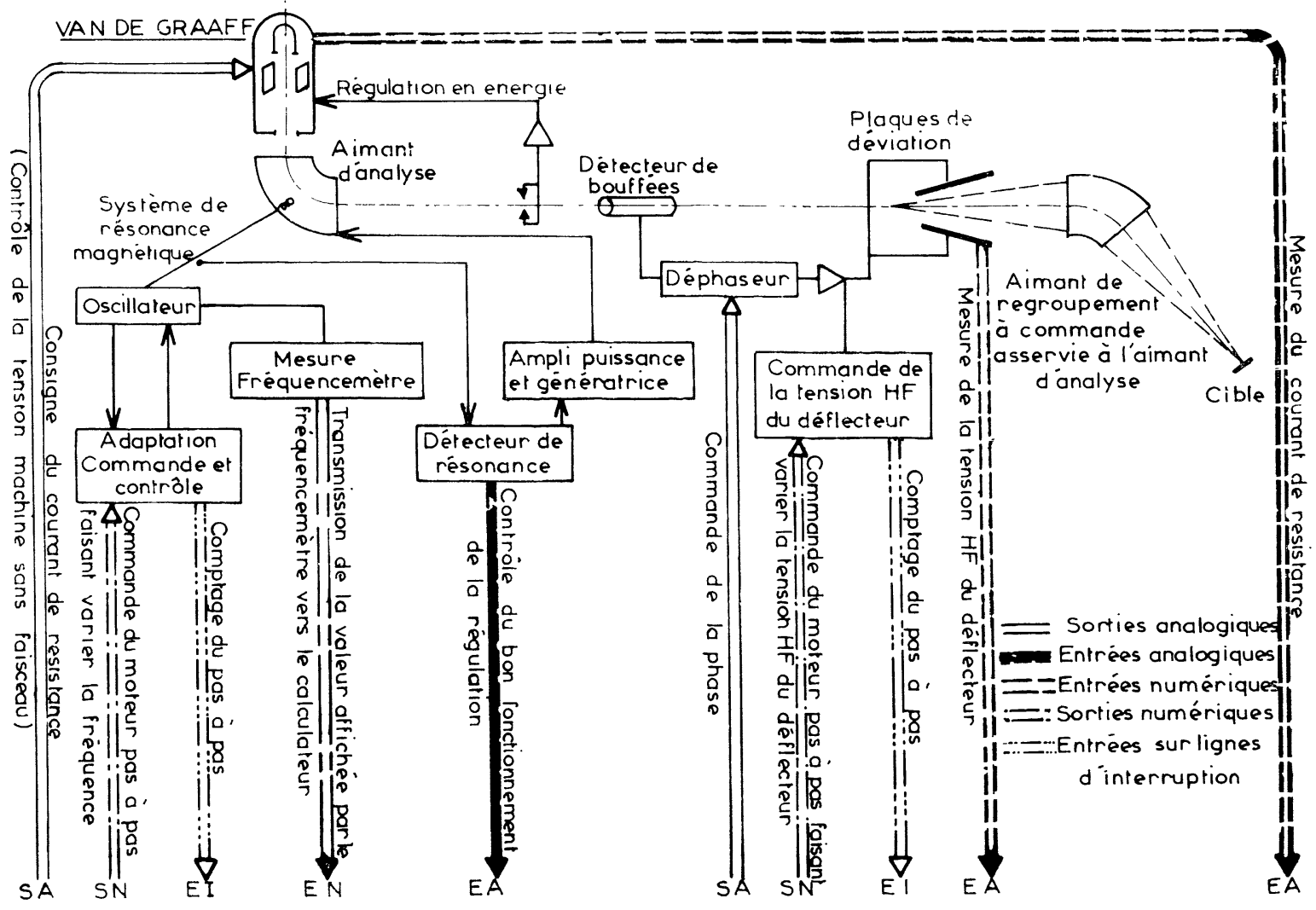

FIG. 1. - Conduite de l'accélérateur. 
de 24 bits, 4 lignes de sorties à 24 relais, 24 relais de scrutation analogique, trois sorties analogiques, $18 \mathrm{li}$ gnes d'interruption et une unité de visualisation.

II. Conduite de l'accélérateur. - L'énergie et les grandeurs liées sont les seuls paramètres contrôlés dans un premier temps. La régulation d'énergie est asservie au champ magnétique d'analyse, lui-même contrôlé par la fréquence d'un circuit de résonance nucléaire [2]. Le rôle du calculateur est de rendre et maintenir cette fréquence égale à la valeur de consigne. Les expériences de temps-de-vol nécessitent un dispositif de regroupement de bouffées d'ions dont le principe est rappelé par la figure 1. Le calculateur doit commander dans ce dispositif la haute tension continue alimentant l'amplificateur HF de puissance et le déphasage entre la tension sinusoïdale issue du détecteur de bouffées et la tension appliquée aux plaques de déflexion [3].

III. Réduction des données. Commande de l'expérience. Programme " Etoile ". - Nous avons opté pour un fonctionnement en temps différé en accumulant pendant chaque séquence d'acquisition les résultats dans différents types de mémoires intermédiaires (sélecteurs et échelles).

Lorsque le temps de comptage est suffisant, le contenu de ces mémoires intégratrices est acquis en mémoire centrale et édité sous une forme appropriée sur les supports extérieurs choisis. Cette façon de faire présente l'avantage de libérer complètement, pendant chaque cycle d'acquisition, le calculateur pour différentes tâches : $i$ ) sorties lentes concernant le cycle précédent; ii) dépouillement des résultats concernant ce même cycle; iii) éventuellement travaux de mises au point de programme ou calculs scientifiques divers.

Entre deux cycles, il peut être nécessaire de modifier certains paramètres de l'expérience. Nous commandons par le calculateur : i) un porte-échantillon à trois positions; ii) la position angulaire d'un détecteur ou d'un ensemble de détecteurs; iii) l'alimentation en courant d'un aimant de précession du spin des neutrons (mesures de polarisation).

Étoile, un programme écrit en langage Symbol 90-10, assure l'exécution de ces fonctions, à l'exclusion du dépouillement. Il est opérationnel depuis plus d'un an et a rendu de grands services; néanmoins il n'est pas très souple, encombrant en mémoire, par conséquent difficile à compléter pour assurer d'autres fonctions et enfin il ne permet pas de faire du dépouillement « au fur et à mesure » en automaticité complète.

IV. " Fortran " et moniteur. - Pour pallier ces défauts, nous avons développé une bibliothèque complète de sous-programmes de commande et de lecture de l'appareillage en lignes, appelables en Fortran. Le physicien peut ainsi organiser d'une façon extrêmement souple toute sa manipulation. Un programme principal appelle alternativement les sousprogrammes de commande et de contrôle et le sousprogramme de dépouillement. Il s'agit donc là d'une véritable « console en software ». Le programme principal se réduit à une suite d'appels et d'instructions de comparaisons entre quantités désirées et quantités mesurées; il est le reflet logique exact de ce que serait l'activité du physicien en l'absence de calculateur (ordres pour régler l'appareillage, lectures d'appareils et décisions en fonction de ces lectures).

Enfin, pour gagner non seulement en souplesse d'emploi mais en encombrement mémoire, nous utilisons un moniteur d'enchaînement de travaux écrit par le Département de Calcul Électronique du C.E.A. Ce programme appelle, par la lecture d'une « table tampon » réalisée sur cartes perforées, ou par dialogue à la machine à écrire, tout programme figurant sur une bande magnétique de gestion. On charge ainsi alternativement les programmes de contrôle, commande et acquisition, et les programmes de dépouillement. Les programmes peuvent figurer sur la bande sous différentes versions, offrant ainsi toute une gamme de possibilités entre l'automaticité complète ou au contraire la mise en œuvre de quelques fonctions particulières. Par exemple, un sigle frappé à la machine à écrire provoque la lecture et l'édition sur table traçante du spectre contenu dans tel sélecteur, ou au contraire la constitution d'une table tampon adéquate permet de lancer pour plusieurs heures une manipulation bien au point, avec contrôle de l'accélérateur et des expériences, acquisitions, et calculs des grandeurs physiques, avec, éventuellement suivant la qualité des résultats des prises de décision automatiques quant à la suite du déroulement des mesures.

\section{BIBLIOGRAPHIE}

[1] Bluet (J. C.), Leroy (J. L.), Anciaux (J. F.), ARnaud (A.) et FARdeau (P.), Onde électr., 1967, no 448 bis, 1395.

[2] Winter (S. D.), Onde électr., 1955, no 334, 995.

[3] Szabo (I.), Onde électr., 1966, no 472, 860. 